\title{
Estimation of Thermal Destruction of AuGeNi Ohmic Contacts of Resonant Tunneling Diodes on the Basis of Nanoscale AIAs/GaAs Heterostructures
}

\author{
Mstislav O. Makeev, Yury A. Ivanov, Sergey A. Meshkov, Vladimir Y. \\ Sinyakin, Anton I. Ivanov \\ Bauman Moscow State Technical University, Moscow, Russia
}

\begin{abstract}
Keywords: Resonant tunneling diode, AuGeNi ohmic contacts, destruction, thermal impact,
\end{abstract} degradation processes, contact resistance, nonlinear radiosignal transmitters.

\begin{abstract}
In the present work the thermal destruction of AuGeNi ohmic contacts of resonant tunneling diodes using artificial aging was researched. The functional dependence of contact resistance of AuGeNi ohmic contacts of resonant tunneling diodes on time and temperature was put forward. This dependence is valid in case the temperatures are lower than $300^{\circ} \mathrm{C}$, it was determined for two parties of resonant tunneling diodes. Obtained results can be used for reliability prediction of resonant tunneling diodes and radio-electronic devices on its basis.
\end{abstract}

\section{Introduction}

One of the ways to improve the quality of radio-electronic systems is the utilization of semiconductor devices based on the quantum-size effects of charge transport. Such devices are resonant tunneling diodes (RTD) based on the multilayered nanoscale AlGaAs/GaAs resonant tunneling heterostructures with transverse charge transport. Small tunneling time of electrons provides extremely high performance of the diode. Nonlinearity of RTD is conserved up to $10 \mathrm{THz}[1,2]$.

Changing the parameters of heterostructure layers (thickness, elemental composition)can possibly contribute to varying the shape of a current-voltage (IV) curve. These properties of RTD allow producing different nonlinear radiosignal transmitters on its basis: mixers, rectifiers, multipliers and generators for super high frequency (SHF) and extremely high frequency (EHF) ranges. Functional characteristics of such devices can be improved by means of optimization of nonlinear element IV curve shape [3-5]. The extensive bibliography is devoted to the research of RTD properties, while reliability of RTD is poorly studied.

A number of works are devoted to the reliability of RTD and nonlinear radiosignal transmitters on its basis where reliability of RTD is studied based on the research of RTD heterostructure thermal destruction processes due to elemental interdiffusion [6,7]. It was shown, how degradation processes impact on the IV curve shape of RTD and the parameters of the radiosignal mixers based on RTD. This work is the continuation of reliability research of RTD, and the aim of this work is to study thermal destruction processes of AuGeNi ohmic contacts of RTD.

\section{Experimental}

To solve the problem, posed by the authors of this paper, the technique for determination dependence of contact resistance RC of the ohmic contacts on time and temperature was developed. Block diagram of this technique is shown in Fig. 1.

Experimental research was carried out for the two parties of RTD produced on different factories. In batch №1 there were twenty RTD, in batch №2 - thirty-two.

For experimental research, the technique of accelerated aging by means of the thermal impact on the sample was used. The temperature of impact was $300^{\circ} \mathrm{C}$. At this temperature, the failure mechanisms are the same as on normal operating conditions [8]. 
The thermal impact on RTD was carried out using laboratory air thermostat, which provides to vary the temperature in the range from 40 to $300{ }^{\circ} \mathrm{C}$ with accuracy $\pm 5{ }^{\circ} \mathrm{C}$. Total time of thermal impact for batch №1 was 150 hours and for batch №2 - 27 hours.

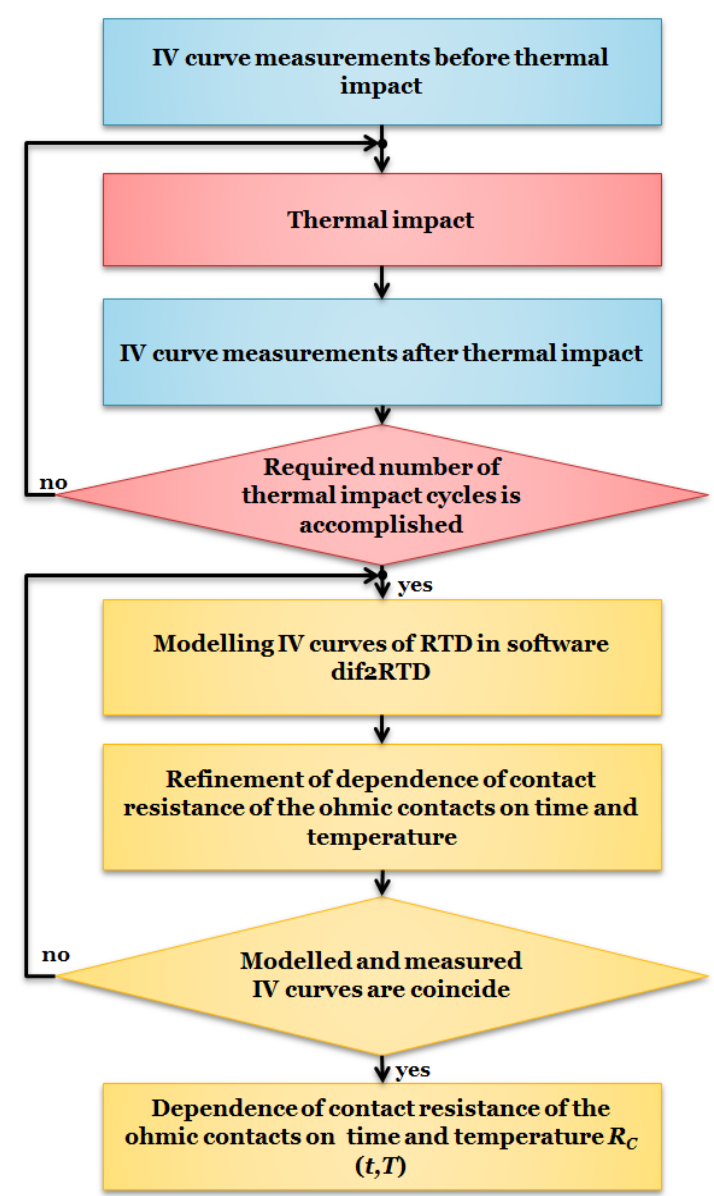

Fig. 1. Block diagram of the technique for the determination of dependence of the contact resistance of AuGeNi ohmic contacts on time and temperature.

IV curve measurements of RTD before and after thermal impact were performed using microprobe unit, which consists of a microprobe device, power supply Agilent E3641A and personal computer (PC). This unit provides IV curve measuring of the diodes in voltage range from 0 to $36 \mathrm{~V}$ (accuracy $\Delta \mathrm{U}= \pm 1 \mathrm{mV}$ ) and current range from 0 to $1 \mathrm{~A}$ (accuracy $\Delta \mathrm{I}= \pm 10 \mu \mathrm{A}$ ).

\section{Results and Discussion}

In accordance with the developed technique, IV curves of RTD of batch №1 and batch №2 were measured, and the statistic processing was performed. Dispersion of IV curves lies within the measurement error range. Therefore, in Fig. 2 IV curves, averaged over whole batch are shown.

In [7,9] it was indicated, that diffusion blur in the resonant tunneling structure affects RTD IV curve shape. Modelling of the diffusion processes for the selected parameters of thermal impact with the means of software calculation complex dif2RTD [10], developed by the authors of this work, indicates that the diffusion blur of the researched resonant tunneling structure is negligibly small and does not change significantly RTD IV curve shape. Diffusion blur of Si in the region near the contacts also has no significant effect on the contact resistance and IV curve of RTD. To explain the detected shift in RTD IV curves one can suggest that AuGeNi ohmic contacts are exposed by degradation as a results of thermal impact which causes an increase of their contact resistance $R_{C}$.

AuGeNi ohmic contacts were developed by Braslau [11]. These contacts have made a good showing while producing devices based on GaAs because of their small contact resistivity $\left(10^{-6}-10^{-8} \mathrm{Ohm} \cdot \mathrm{cm}^{2}\right)$, and good reproducibility while manufacturing. Moreover, they can be 
readily incorporated into a technological process. At the same time, these contacts have a sufficiently low thermal stability and a high surface roughness [12-15]. This fact may result in the reduction of a device reliability in general.

Ohmic contacts of the researched RTD had a structure: n-GaAs/Au+Ge/Ni/Au. Technological process of producing AuGeNi ohmic contacts includes the following steps: sequential deposition of the $\mathrm{Au}-\mathrm{Ge}, \mathrm{Ni}$ and $\mathrm{Au}$ layers, and, finally, annealing of the structure.
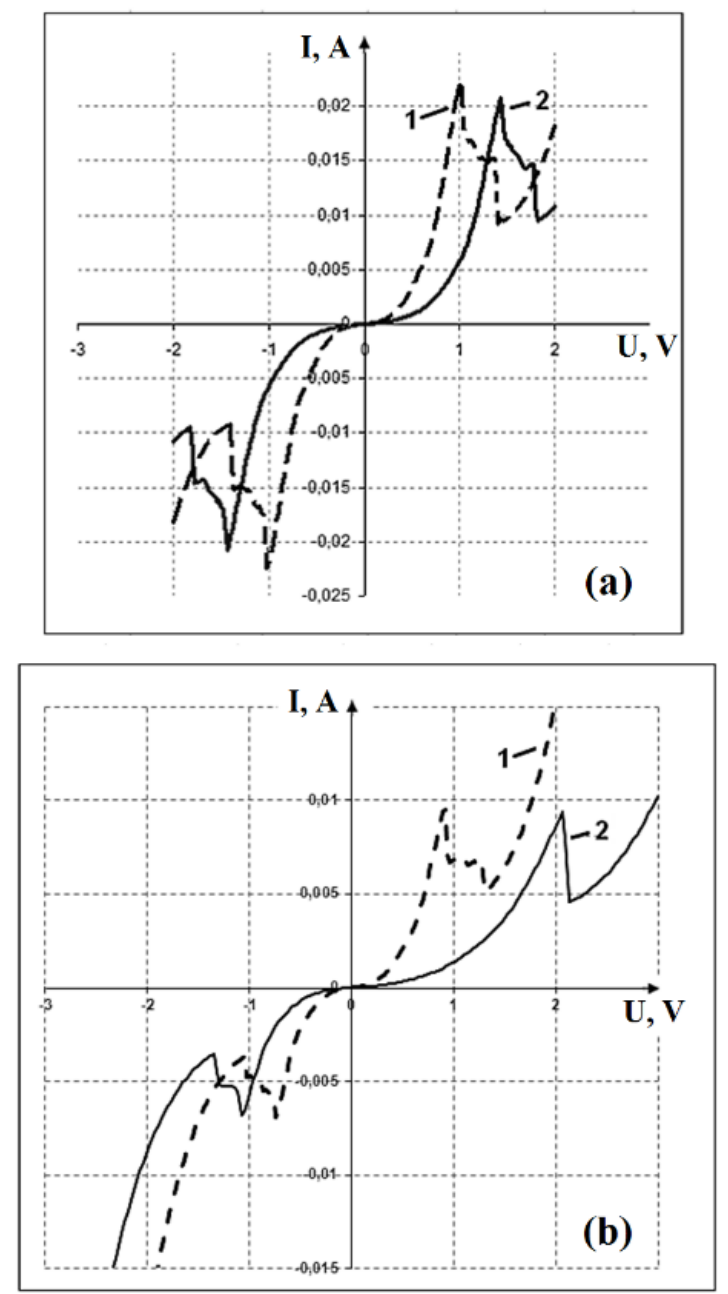

Fig. 2. Experimental IV curves of RTD for batch №1 (a) and batch №2 (b) before and after thermal impact (1 - before, 2 - after 150 (a) and 27 (b) hours).

In the process of ohmic contacts thermal treatment, Ga diffuses from the GaAs substrate, wherein $\mathrm{Au}$ interact with $\mathrm{Ga}$ forming the layer of $\beta$-AuGa phase. The eutectic temperature of suchan alloy $(\mathrm{Au}-\mathrm{Ge})$ is $360^{\circ} \mathrm{C}$. Ge diffuses from the $\mathrm{Au}-\mathrm{Ge}$ alloy into the top layer of $\mathrm{Ni}$ at the temperature of $350{ }^{\circ} \mathrm{C}$ (and more), forming $\mathrm{Ni}_{3} \mathrm{Ge}$ layer. However, only a small portion of $\mathrm{Ge}$ remains in the first layer of Au deposited on the GaAs substrate, and Ni reacts with GaAs forming $\mathrm{Ni}_{\mathrm{x}} \mathrm{GaAs}$. The next step is a forming of $\mathrm{NiAs}(\mathrm{Ge})$-layer at the temperature of $450{ }^{\circ} \mathrm{C}$ as a result of $\mathrm{Ge}$ penetration in $\mathrm{Ni}_{\mathrm{x}} \mathrm{GaAs}$ and the replacement of $\mathrm{Ga}$. The contact acquires $\beta$ $\mathrm{AuGa} / \mathrm{NiAs}(\mathrm{Ge}) / \mathrm{GaAs}$ structure (see Fig. 3), and cooling leads to the forming of a new highly doped by Ge layer of GaAs $[13,16]$. It results in the narrowing of potential barrier at the metalsemiconductor interface.

Thus, Ge is a doping element, which provides the possibility of tunneling through the potential barrier. $\mathrm{Ni}$ is a catalyzer: it interacts with As, accelerating incoming diffusion of $\mathrm{Ga}, \beta$-AuGa-layer formation and doping of GaAs by Ge. Furthermore, Ni improves the uniformity of the contact. Au serves as a metallization and contributes the creating of Ga vacancies.

$\mathrm{NiAs}(\mathrm{Ge})$-layer is mainly responsible for the electrical properties of $\mathrm{AuGeNi}$ ohmic contacts. It has a grain structure with the grain size about $\sim 1 \mu \mathrm{m}$ in width and about $\sim 250 \mathrm{~nm}$ in depth. It was 
found out, that the larger the contact layer area of the $\mathrm{NiAs}(\mathrm{Ge})$ with $\mathrm{GaAs}$ substrate is the lower is the contact resistivity $\rho_{c}$ which suggests the importance of a direct contact of $\mathrm{NiAs}(\mathrm{Ge})$-layer with GaAs [12,13]. On this basis, the optimal structure of AuGeNi ohmic contacts will consist of a GaAs substrate completely covered by $\mathrm{NiAs}(\mathrm{Ge})$-layer, and a uniform $\beta$-AuGa-layer, located on the top of $\mathrm{NiAs}(\mathrm{Ge})$ with no contact with GaAs substrate.

On the one hand, $\beta$-AuGa-layer also plays an important role in producing ohmic contacts with low $R_{c}$. On the other hand, this layer is responsible for the thermal instability of AuGeNi ohmic contacts. Degradation of such ohmic contacts has the following mechanism: besides blurring of the $\beta$-AuGa-layer the interdiffusion of $\mathrm{Au}$ and $\mathrm{Ga}$ takes place and, therefore, new regions of $\beta$-AuGa are formed. It results in a decrease of GaAs substrate area covered by $\mathrm{NiAs}(\mathrm{Ge})$ and, therefore, in the increase of contact resistance $R_{c}$.

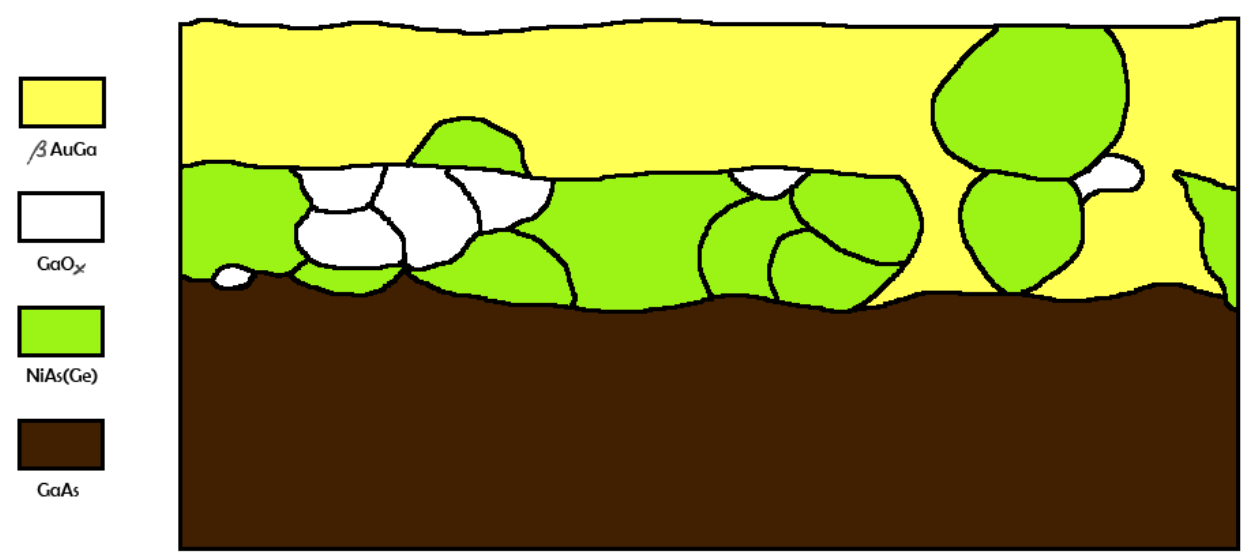

Fig. 3. Cross-section of the AuGeNi ohmic contacts after thermal annealing.

On the assumption of AuGeNi contact degradation occurs by means of a diffusion blur, variation of ohmic resistance $R_{C}$ in time can be described as [17]:

$$
R_{C}=R_{C 0}+\gamma \cdot \exp \left(-\frac{E_{a}}{2 k T}\right) \cdot \sqrt{t}
$$

where $R_{C 0}$ - initial contact resistance (after the production), Ohm. For the contact area $\mathrm{S}_{C}=25 \mu \mathrm{m}^{2}$ at the value of the contact resistivity about $1 \cdot 10^{-6} \mathrm{Ohm} \cdot \mathrm{cm}^{2}[12,16] R_{C 0}$ equals to $4 \mathrm{Ohm} ; \gamma-$ coefficient, $\mathrm{Ohm} / \mathrm{s}^{1 / 2} ; E_{a}$ - activation energy, eV. Activation energy $E_{a}$ of degradation phenomena of AuGeNi ohmic contacts equals $1 \mathrm{eV}[18,19] ; T$ - temperature, $\mathrm{K} ; k$ - Boltzmann constant $\left(k=8,61710^{-5} \mathrm{eV} \cdot \mathrm{K}^{-1}\right) ; t$ - time, $\mathrm{s}$.

According to the developed technique, coefficient $\gamma$ was determined. It characterizes the velocity of ohmic contact diffusion degradation and included in equation (1) - dependence of contact resistance of the AuGeNi ohmic contacts on time and temperature. For batch №1 coefficient $\gamma$ was $408,2 \mathrm{Ohm} / \mathrm{s}^{1 / 2}$ and for batch №2 was $25 \mathrm{Ohm} / \mathrm{s}^{1 / 2}$.

Thus, the functional dependence of AuGeNi ohmic contact resistance on time and temperature for batch №1 is expressed as:

$$
R_{C}(T, t)=4+408,2 \cdot \exp \left(-\frac{1}{2 k T}\right) \cdot \sqrt{t},
$$

and for batch №2:

$$
R_{C}(T, t)=4+25 \cdot \exp \left(-\frac{1}{2 k T}\right) \cdot \sqrt{t} .
$$

The coefficients $\gamma$, which show the degradation velocity of ohmic contacts, vary more than 16 times. Based on these special cases, one can conclude, that coefficient $\gamma$ is susceptible to the 
construction and ohmic contact manufacturing technology changes. It has a direct relationship to the reliability of devices with such contacts while contact resistance increase leads to the shift of IV curve of the diode and, therefore, to the degradation failure.

\section{Conclusions}

Functional dependence of contact resistance $R_{\mathrm{C}}$ of the ohmic contacts was proposed. This dependence connects construction of an ohmic contact and its producing technology as well as duration $t$ and temperature $T$ of operating with $R_{\mathrm{C}}$ changing. Coefficient $\gamma$ which is included in this dependence characterizes the degradation velocity of ohmic contacts and can be used for assessing the AlAs/GaAs RTD production quality.

The dependence $R_{\mathrm{C}}(t, T)$ was experimentally determined for the two RTD batches with AuGeNi ohmic contacts (coefficients $\gamma$ of ohmic contacts were 25 and 408,2 Ohm/ $\mathrm{s}^{1 / 2}$ for batch №1 and for batch №2, respectively). These values can be used for RTD reliability prediction and radiotechnical devices on its basis.

\section{Acknowledgements}

The research work was supported by Ministry of Education and Science of the Russian Federation under state task № 16.1116.2014/K.

\section{References}

[1] N.V. Alkeev, S.V. Averin, A.A. Dorofeev, N.B. Gladysheva and M.Yu. Torgashin: Russ. Microelectronics Vol. 39 (2010), p. 331.

[2] W.R. Frensley: Appl. Phys. Lett. Vol. 51 (1987), p. 448.

[3] V.N. V'juginov, A.G. Gudkov, V.A. Dobrov, S.A. Meshkov and V.V. Popov: Elektromagnitnye volny i elektronnye sistemy Vol. 1 (2012), p. 4.

[4] Y.A. Ivanov, S.A. Meshkov, I.A. Fedorenko, N.V. Fedorkova and V.D. Shashurin: J. Commun. Technol. El. Vol. 55 (2010), p. 982.

[5] Y.A. Ivanov, S.A. Meshkov, V.Y. Sinjakin, I.A. Fedorenko, N.V. Fedorkova, I.B. Fedorov and V.D. Shashurin: Nanoengineering Vol. 1 (2011), p. 34.

[6] M.O. Makeev, Y.A. Ivanov and S.A. Meshkov: Nanoengineering Vol. 4 (2011), p. 44.

[7] M.O. Makeev, Y.A. Ivanov, V.Y. Sinyakin, S.A. Meshkov, S.V. Agasieva and V.D. Shashurin: CriMiCo 2014 - 2014 24th International Crimean Conference Microwave and Telecommunication Technology, Conference Proceedings (2014), p. 754.

[8] M.I. Gorlov and A.V. Strogonov: Peterburg electronic journal Vol. 4 (1996), p. 35.

[9] M.O. Makeev, Y.A. Ivanov, S.A. Meshkov and V.D. Shashurin: Science and education: electronic scientific and technical journal Vol. 11 (2013), p. 229.

[10]M.O. Makeev, Y.A. Ivanov, S.A. Meshkov, Y.N. Litvak, D.E. Migal and V.V. Nazarov: Nanoengineering Vol. 1 (2014), p. 24.

[11]N. Braslau, J.B. Gunn and J.L. Staples: Solid-State Electron. Vol. 10 (1967), p. 381.

[12]M. Murakami: Science and Technology of Advanced Materials Vol. 3 (2002), p. 1.

[13] Y.C Shih, M. Murakami, E.L. Wilkie and A.C. Callegari: J. Appl. Phys. Vol. 62 (1987), p. 582.

[14]T.K. Higman, M.A. Emanuel, J.J. Coleman, S.J. Jeng and C.M. Wayman: J. Appl. Phys. Vol. 60 (1986), p. 679. 
[15] K.C. Lee and J. Res. Natl. Inst. Stand. Technol. Vol. 103 (1998), p. 177.

[16]T.V. Blank and Yu.A. Gol'dberg: Semiconductors Vol. 41 (2007), p. 1263.

[17]M.O. Makeev, Y.A. Ivanov, S.A. Meshkov and V.V. Nazarov: Science and education: electronic scientific and technical journal Vol. 9 (2012), p. 14.

[18] V.A. Vashchenko, V.F. Sinkevitch: Physical limitations of semiconductor devices (Springer, 2008).

[19]J.W. Dilorenzo, D.D. Khandelwal. GaAs FET Principle and Technology (Washington, Artech House, 1982). 